\title{
Theoretical simulation of different 3D separator geometries for lithium-ion batteries
}

Cite as: AIP Conference Proceedings 2293, 260005 (2020); https://doi.org/10.1063/5.0026447

Published Online: 25 November 2020

D. Miranda, R. Gonçalves, F. Miranda, A. M. Almeida, C. M. Costa, and S. Lanceros-Méndez

ARTICLES YOU MAY BE INTERESTED IN

Cone geometry optimization and thermal behavior for lithium-ion battery separators

AIP Conference Proceedings 2293, 260006 (2020); https://doi.org/10.1063/5.0026453

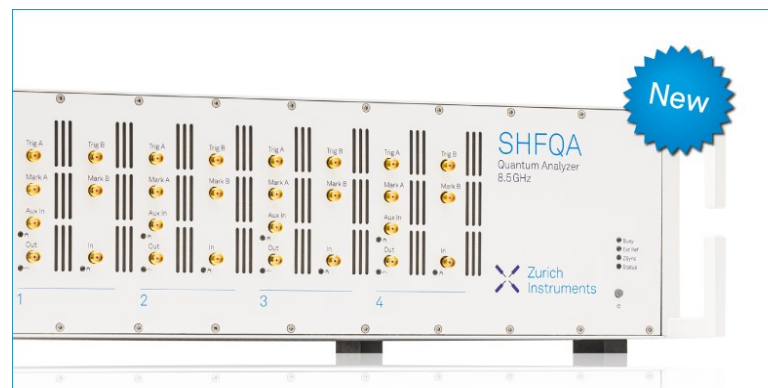

Your Qubits. Measured.

Meet the next generation of quantum analyzers

- Readout for up to 64 qubits

- Operation at up to $8.5 \mathrm{GHz}$,

Find out more

mixer-calibration-fre

- Signal optimization with minimal latency 


\title{
Theoretical Simulation of Different 3D Separator Geometries for Lithium-Ion Batteries
}

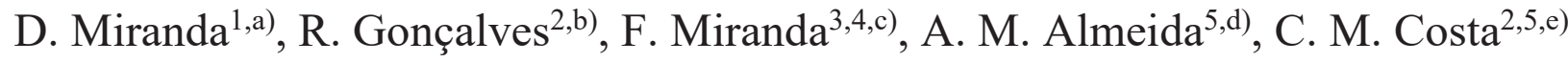 \\ and S. Lanceros-Méndez ${ }^{6,7, f)}$ \\ I2Ai- Polytechnic Institute of Cávado and Ave, Barcelos, Portugal \\ ${ }^{2}$ Centro de Química, Universidade do Minho, 4710-057 Braga, Portugal \\ ${ }^{3}$ CIDMA, Universidade de Aveiro, Aveiro, Portugal \\ ${ }^{4}$ Instituto Politécnico de Viana do Castelo, Viana do Castelo, Portugal \\ ${ }^{5}$ Centro de Física, Universidade do Minho, 4710-057 Braga, Portugal \\ ${ }^{6}$ BCMaterials, Basque Center for Materials, Applications and Nanostructures, UPV/EHU Science Park, 48940 \\ Leioa, Spain \\ ${ }^{7}$ IKERBASQUE, Basque Foundation for Science, 48013 Bilbao, Spain \\ a)Corresponding author: damiranda@ipca.pt \\ b)r.goncalves@quimica.uminho.pt \\ c)fmiranda@estg.ipvc.pt \\ d)coimbra@fisica.uminho.pt \\ e)cmscosta@fisica.uminho.pt \\ f)senentxu.lanceros@bcmaterials.net
}

\begin{abstract}
The battery separator is an essential component of batteries and affects their cycling performance. In this work, the effect of different 3D geometries of the battery separator on battery performance was studied keeping the same volume. It was observed that the different geometries affect the cycling performance, the best geometry being the perforated one that cycled up to $90 \mathrm{C}$. The cycling performance is affected by parameters such as the separator thickness and the electrolyte volume. Through the control of the battery separator geometry it is possible to obtain high performance lithium-ion batteries.
\end{abstract}

Keywords: Separator, simulation, capacity, lithium ion battery.

PACS: 88.80. F-, 88.80.ff, 88.05.-b

\section{INTRODUCTION}

Electrochemical energy storage devices, such as lithium ion batteries, are needed based on the fact that the obtained electricity from different sources should be stored in portable devices and electric vehicles [1]. Lithium batteries were introduced in the market in 1992 by Sony and stand out among other energy storage devices due to being lighter, cheaper, showing higher energy and power density, no memory effect, prolonged service-life, environmental friendliness, self-discharge and higher number of charge/discharge cycles [1]. They are composed by electrodes and the separator in which this last component is essential to control the diffusion and lithium ion numbers during the charge-discharge behavior [2].

The battery separator is placed between the electrodes and several parameters determine its performance such as morphology (porosity and pore size), wettability, thermal stability, mechanical properties and chemical compatibility [2].

Three-dimensional (3-D) batteries are beneficial compared with 2-D batteries due to the higher energy storage and the reduction of the length in the diffusion paths [3]. 3-D microbatteries have been obtained by fused-filament fabrication (FFF) in which it is possible obtained batteries with free form-factor with excellent performance [4].

The separator component is basically a robust element, with a porous morphology, in which 3D-structures are being studied [5].The influence of battery separators with patterned surfaces, including arrays of hexagons, lines, zig-zags and pillars, in battery performance has been evaluated and demonstrated that the structure influences

International Conference of Numerical Analysis and Applied Mathematics ICNAAM 2019

AIP Conf. Proc. 2293, 260005-1-260005-5; https://doi.org/10.1063/5.0026447

Published by AIP Publishing. 978-0-7354-4025-8/\$30.00 
battery performance, depending on different parameters such as contact area between the electrolyte and the separator, as it affects ion diffusion [5].

3D-structures for separators have been rarely investigated for lithium-ion batteries and the aim of this work is to evaluate the influence of the different 3D separator structures, while maintaining the same volume, through theoretical simulation, as shown in figure 1.

\section{METHODOLOGY}

The theoretical simulation was performed by the Finite Element Method, applying the Doyle/Fuller/Newman model that describes the battery operation [6].

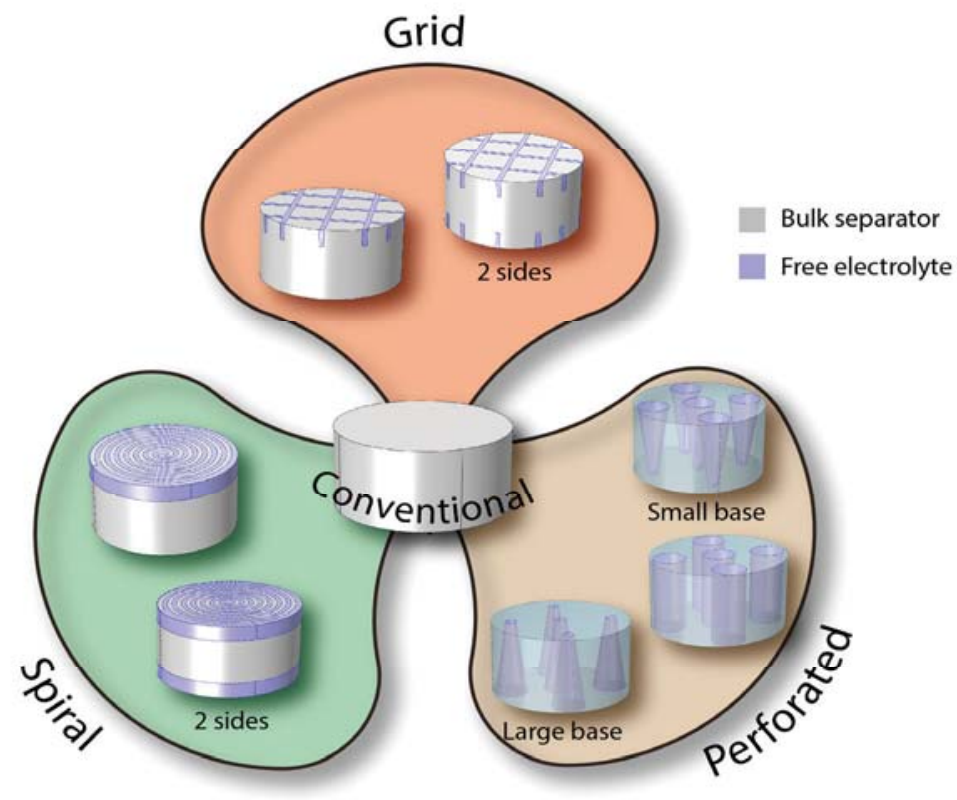

FIGURE 1. Schematic representation of different porous separator geometries (spiral, grid, spiral 2 sides, grid 2 sides, large base funnel, small base funnel, perforated and conventional) applied in the simulated batteries.

Table 1 presents the main values of the different parameters used in the simulations for the separator and the cathode in $\mathrm{Li} / \mathrm{Li}_{x} \mathrm{FePO}_{4}$ half cells. The complete mathematical formulation of the simulations can be found in [6].

TABLE 1. Specific parameters used in the developed simulations.

\begin{tabular}{|c|c|c|c|}
\hline \multicolumn{4}{|c|}{ Electrochemical parameters of the components } \\
\hline Parameter & Unit & Separator & Cathode $\left(\mathrm{Li}_{x} \mathrm{FePO}_{4}\right)$ \\
\hline$C_{E, i, 0}$ & mol.m $\mathrm{m}^{-3}$ & & 3900 \\
\hline$C_{E, i, \max }$ & mol.m $\mathrm{m}^{-3}$ & & 21190 \\
\hline$C_{L}$ & mol.m $\mathrm{m}^{-3}$ & 1000 & \\
\hline$L_{i}$ & $\mathrm{~m}$ & $150 \times 10^{-6}$ & $20 \times 10^{-6}$ \\
\hline$k_{i}(T)$ & S.m $\mathrm{m}^{-1}$ & a) & a) \\
\hline$D_{i}(T)$ & $\mathrm{m}^{2} \cdot \mathrm{s}^{-1}$ & b) & b) \\
\hline$D_{e f, i}$ & $\mathrm{~m}^{2} \cdot \mathrm{s}^{-1}$ & $D_{i}(T) \times 4.84 \times 10^{-2}$ & $D_{i}(T) \times 0.444^{1,5}$ \\
\hline$D_{L I}$ & $\mathrm{~m}^{2} \cdot \mathrm{s}^{-1}$ & & $3.2 \times 10^{-13}$ \\
\hline$D_{L I}(T)$ & & & c) \\
\hline$\varepsilon_{i}$ & & 0.70 & 0.444 \\
\hline$\sigma_{l}$ & S.m $\mathrm{m}^{-1}$ & & 11.8 \\
\hline$i_{l C}$ & A.m $m^{-2}$ & 17.5 & \\
\hline$E_{a d, i}$ & J.mol-1 & & $39 \times 10^{3}$ \\
\hline$E_{a k, i}$ & J.mol-1 & & $29 \times 10^{3}$ \\
\hline$T$ & K & 298.15 & 298.15 \\
\hline
\end{tabular}


a) Model Fits: Ionic conductivity as a function of salt concentration in the $\mathrm{LiPF}_{6}$ in EC:DMC mixture [6]:

$k i(T)=c \times\left(-10.5+(0.0740 \times T)-\left(\left(6.9610^{-5}\right) \times\left(T^{2}\right)\right)+(0.668 \times c)-(0.0178 \times c \times T)\right.$

$\left.+\left(\left(2.8 \times 10^{-5}\right) \times c \times\left(T^{2}\right)\right)+\left(0.4949 \times c^{2}\right)-\left(\left(8.86 \times 10^{-4}\right) \times\left(c^{2}\right) \times\left(T^{2}\right)\right)\right)^{2}$

b) Diffusion coefficient as a function of temperature [6]

$D_{i}(T)=10^{\left(-(0.22 c)-4.43-\left(\left(\frac{54}{T-229-5 c}\right)\right)\right)}$

c) Diffusion coefficient of Li ions [6]

$D_{L i}(T)=D_{t 298,15 i} e^{-E_{a d i} / R}\left(\frac{1}{T}-\frac{1}{298.15}\right)$

\section{RESULTS AND DISCUSSION}

$\mathrm{Li} / \mathrm{Li}_{\mathrm{x}} \mathrm{FePO}_{4}$ half-cell lithium-ion batteries with different porous separator geometric configurations (spiral, spiral 2 sides, grid, grid 2 sides, large base funnel, small base and perforated), as illustrated in figure 1, were simulated at low, medium and high discharge rates with the same volume of its components and compared to the conventional geometry as shown in figure 2.

Figure 2 shows that at low discharge rates up to $20 \mathrm{C}$, the discharge value is very similar for all geometries due to the low effective ion mobility at this discharge rate, which does not affect the intercalation process for the different geometries.

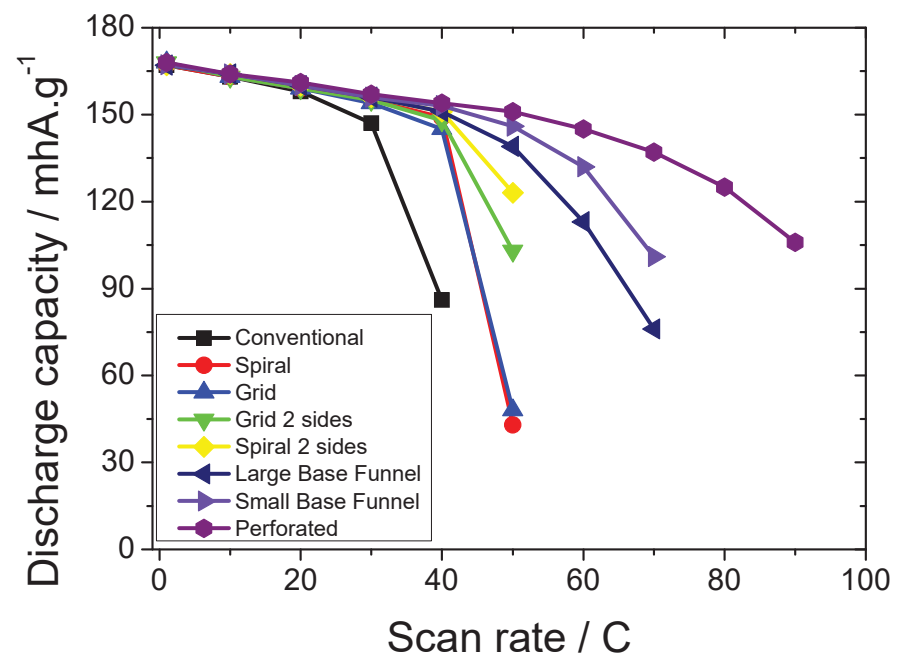

FIGURE 2. Delivered discharge capacity as a function of scan rate for the different separator geometries.

For discharge rates above $30 \mathrm{C}$, different discharge capacity values are observed for the different geometries in which the conventional geometry is very limited operating up to $40 \mathrm{C}$ with a discharge capacity value of $86 \mathrm{mAh} \cdot \mathrm{g}^{-1}$.

The spiral, spiral 2 sides, grid, grid 2 sides geometries operate up to $50 \mathrm{C}$ with discharge capacity values of 43 $\mathrm{mAh} \cdot \mathrm{g}^{-1}, 123 \mathrm{mAh} \cdot \mathrm{g}^{-1}, 48 \mathrm{mAh} \cdot \mathrm{g}^{-1}$ and $103 \mathrm{mAh} \cdot \mathrm{g}^{-1}$, respectively.

The large base funnel and small base funnel geometries operate up to $70 \mathrm{C}$ with discharge capacity values of 76 $\mathrm{mAh} \cdot \mathrm{g}^{-1}$ and $101 \mathrm{mAh} \cdot \mathrm{g}^{-1}$, respectively. Finally, the best geometry operates up to $90 \mathrm{C}$ and is the perforated with a discharge capacity value of $106 \mathrm{mAh} \cdot \mathrm{g}^{-1}$.

The different discharge capacity values obtained for the different geometries are due to the geometric parameters, such as the free electrolyte volume present in the separator regions (cavities), bulk separator volume, surface areas of contact between free electrolyte with cathode and separator thickness.

At high discharge rates it is required high ion mobility through the separator, where geometries with regions occupied by high volumes of free electrolyte will allow ions to pass through the separator and free electrolyte with high diffusion and ionic conductivity values. 
It is important to note that the thickness of the separator also makes a strong contribution to battery performance, as thicker separators force the ions to travel longer distances until they reach the cathode in the discharge process. These longer distances result into high resistances in ion transport. Although the conventional geometry has the smallest separator thickness (figure 3a)), this battery does not contain regions with high free electrolyte volume (figure 3b)) moving its ions through a bulk separator. This effect reduces battery performance because its ions are subject to low diffusion and conductivity values.

At the discharge rate of $50 \mathrm{C}$, the battery with higher capacity value is the perforated geometry $\left(151 \mathrm{mAh} \cdot \mathrm{g}^{-1}\right)$ in which this geometry presents a high separator thickness. This thickness does not significantly affects the discharge capacity due to the gain associated with the free electrolyte volume that facilitates the diffusion of ions in the discharge process.

The small and large base funnel geometries have the same value of free electrolyte volume and separator thickness, however the contact area between free electrolyte and cathode is larger in the small base funnel. This larger contact area at the electrolyte/cathode interface contributes to easier cathode insertion since the ions show a larger interface region with high diffusion and conductivity implying a higher capacity value obtained at high discharge rates as shown in figure 3.
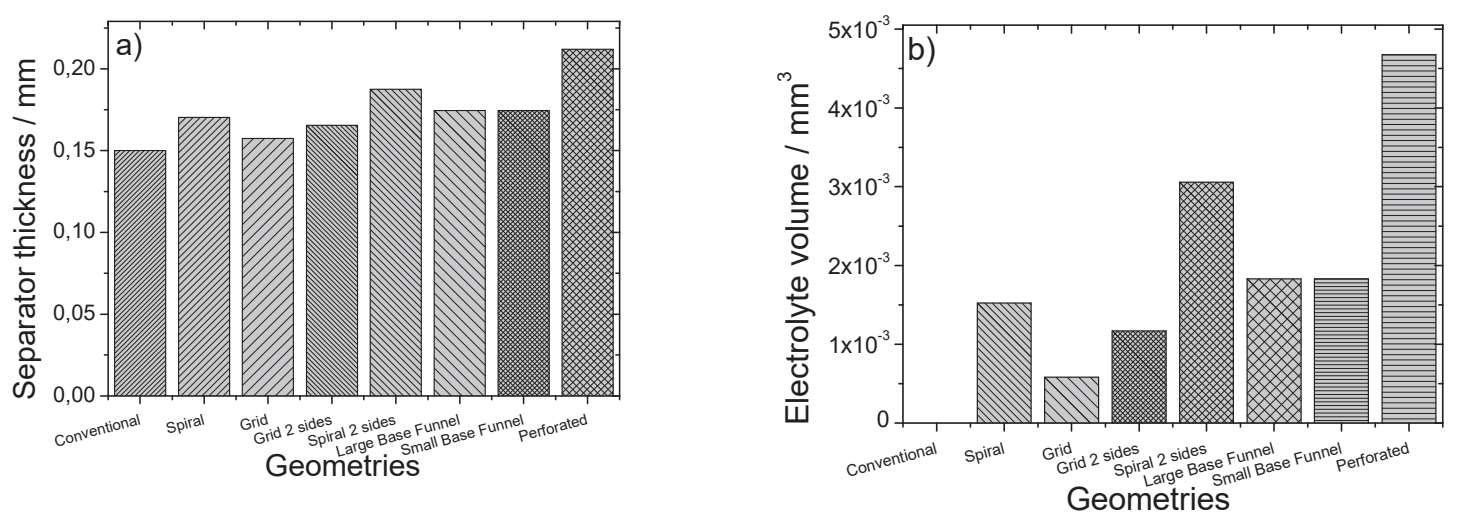

FIGURE 3. a) Separator thickness of different separator geometries, b) Electrolyte volume of the different separator geometries

Finally, batteries with grid and spiral geometries can have configuration on both sides of the separator, as shown in figure 1. In both cases the grid 2 sides and spiral 2 sides' format reach higher capacity values than the batteries with the same geometric configuration present on only one side of the separator (grid and spiral). This is due to the fact that the batteries with geometrical configuration on both sides of the separator have a larger free electrolyte volume (figure $3 \mathrm{~b}$ ) compared to their shape and consequently smaller region thickness corresponding to the bulk separator, as shown in figure 1 which implies a larger region where ions have low diffusion values and ion conductivity.

\section{CONCLUSION}

Battery separator affects e battery performance and, in this work, different 3-D separator geometries were theoretically evaluated on $\mathrm{Li} / \mathrm{Li}_{x} \mathrm{FePO}_{4}$ half-cell lithium-ion batteries with the same volume of the separator. Different 3-D geometries have been shown to improve cycling performance compared to conventional geometry. The discharge capacity value and the maximum discharge rate in which battery operates are affected by the geometry. The high discharge value is obtained for the perforated geometry and operates up to $90 \mathrm{C}$. Geometrical parameters such as separator thickness and electrolyte volume are relevant for determining battery performance and the balance of both parameters is essential for obtaining high discharge capacity. Thus, the presented 3-D geometries enhance the cycling performance of the lithium-ion battery. 


\section{ACKNOWLEDGMENTS}

Portuguese Foundation for Science and Technology (FCT): UID/FIS/04650/2019, PTDC/CTM-ENE/5387/2014, UID/CTM/50025/2013, PTDC/FIS-MAC/28157/2017, grant SFRH/BPD/112547/2015 (C.M.C.) and contract CEECIND/00833/2017 (R.G.), including FEDER funds through the COMPETE 2020 Programme and National Funds through FCT. Financial support from the Basque Government Industry Department under the ELKARTEK and HAZITEK programs is also acknowledged. F. Miranda was supported by the Portuguese Foundation for Science and Technology (FCT - Fundação para a Ciência e a Tecnologia), through CIDMA - Center for Research and Development in Mathematics and Applications, within project UID/MAT/04106/2019.

\section{REFERENCES}

1. M. K. Gulbinska, Lithium ion batteries materials and engineering: Current Topics and Problems from the Manufacturing Perspective, Springer 2014.

2. C. M. Costa, et al., Energy storage materials, In press (2019).

3. J. G. Werner, et al., Energy Environmental Science, 11 (2018) 1261.

4. H. Ragones, et al., Sustainable Energy \& Fuels, 2 (2018) 1542-1549.

5. C. M. Costa, et al., Energy storage materials, In press (2019).

6. M. Doyle, et al., J. Electrochem. Soc. 143 (1996) 1890-1903. 